03

\title{
Оценки пироэлектрических коэффициентов нитридов алюминия и галлия
}

\author{
() С.Ю. Давыдов
}

Физико-технический институт им. А.Ф. Иофрфе РАН,

Санкт-Петербург, Россия

E-mail: Sergei_Davydov@mail.ru

Поступила в Редакцию 1 декабря 2021 г.

В окончательной редакции 16 января 2022 г.

Принята к публикации 22 января 2022 г.

Получено аналитическое выражение для пироэлектрического коэффициента $p$ соединений со структурой вюрцита. Для монокристаллов $\mathrm{AlN}$ и $\mathrm{GaN}$ определены значения $p$, удовлетворительно согласующиеся с результатами расчетов из первых принципов. Обсуждается пироэлектрический эффект в однородных эпитаксиальных пленках и гетероструктурах.

Ключевые слова: пироэлектрический коэффициент, структура вюрцита, монокристалл, тонкая пленка, гетероструктура.

DOI: 10.21883/FTT.2022.05.52329.248

1. Известный еще с античных времен пироэлектрический эффект [1] по-прежнему вызывает интерес исследователей [2-4]. Этот эффект состоит в зависимости спонтанной поляризации $P_{s}$ от температуры $T$ и характеризуется полным пироэлектрическим коэффициентом $p(T)=\left(d P_{s} / d T\right)_{\sigma}$, где $\sigma-$ механическое напряжение, и предполагается, что вектор $\mathbf{P}_{s}$ имеет только одну компоненту вдоль оси $\hat{z}$. Основы современной теории пироэлектричества были заложены Борном [5] и Сцигети [6], сегодняшнее состояние теории отражено в статьях [7-9]. В соответствии с [5-9], имеем

$$
\begin{aligned}
p(T) & =p_{1}(T)+p_{2}(T) \\
& =\left(\frac{\partial P_{s}}{\partial T}\right)_{\varepsilon}+\sum_{i}\left(\frac{\partial P_{s}}{\partial \varepsilon_{i}}\right)_{T}\left(\frac{\partial \varepsilon_{i}}{\partial T}\right)_{\sigma},
\end{aligned}
$$

где $\varepsilon-$ упругая деформация с компонентами $\varepsilon_{i}$. Слагаемое $p_{1}(T)$ называют первичным пироэлектрическим коэффициентом (PC) при постоянной внешней деформации, когда объем и форма образца остаются постоянными („clamped-lattice“ pyroelectricity). Второе слагаемое в (1), называемое вторичным РС, отвечает постоянному напряжению и может быть рассчитано как $p_{2}(T)=\sum d_{i j k} c_{j k l m} \alpha_{l m}$, где $\mathbf{d}, \mathbf{c}$ и $\boldsymbol{\alpha}$ - тензоры пьезоэлектрических напряжений, упругих постоянных и коэффициентов теплового расширения [4]. Если считать тензоры $\mathbf{d}, \mathbf{c}$ и $\boldsymbol{\alpha}$ известными, то задача об определении РС сводится к расчету $p_{1}(T)$, который можно представить в виде суммы $p_{1}^{(1)}(T)$ и $p_{1}^{(2)}(T)$, где первый член отвечает модели жестких точечных ионов, а второй член описывает перераспределение электронного заряда, вызванное колебаниями решетки $[8,9]$. В дальнейшем мы будем рассматривать только соединения типа $\mathrm{A}_{N} \mathrm{~B}_{8-N}$, обладающие структурой вюрцита. Пренебрегая $p_{1}^{(2)}(T)$, согласно [8], получим

$$
p_{1}(T)=\frac{4 e Z^{*} u_{T}}{\sqrt{3} a^{2}} .
$$

Здесь $e$ - элементарный заряд, $Z^{*}$ - эффективный поперечный заряд Борна, $u_{T}=d u / d T, u-$ внутренняя деформация. Учтено также, что в идеальной структуре вюрцита $u=3 / 8$ и объем элементарной ячейки, содержащей 4 атома, равен $\Omega=\sqrt{3} a^{2} c / 2$, где $a$ и $c-$ постоянные решетки, ось $c$ совпадает с осью $\hat{z}$. Воспользовавшись результатами работы [8], после ряда преобразований получим

$$
\begin{gathered}
u_{T}=\frac{4 \gamma_{\mathrm{TO}} C_{V}(T)}{3 c^{2} M \omega_{\mathrm{TO}}^{2}}, \\
C_{V}(T)=k_{\mathrm{B}} \sum_{q_{j}}\left(\hbar \omega_{q_{j}} / k_{\mathrm{B}} T\right)^{2} \exp \left(\hbar \omega_{q_{j}} / k_{\mathrm{B}} T\right) n_{q_{j}}^{2},
\end{gathered}
$$

где $\gamma_{\text {то }}=-d \ln \omega_{\mathrm{TO}} / d \ln u-$ постоянная Грюнайзена для поперечной оптической моды $\omega_{\text {то }} \equiv \omega_{\text {то }}(0)$ при волновом векторе $\mathbf{q}=0, C_{V}(T)-$ теплоемкость решетки при постоянном объеме, $n_{q_{j}}=\left[\exp \left(\hbar \omega_{q_{j}} / k_{\mathrm{B}} T\right)-1\right]^{-1}-$ функция распределения Бозе-Эйнштейна, $M-$ приведенная масса, $k_{\mathrm{B}}$ - постоянная Больцмана, индекс $j$ нумерует ветви фононного спектра. Таким образом, $p_{1}^{(1)}(T) \propto u_{T}(T) \propto C_{V}(T)$, что отмечалось в [8-10].

2. Рассмотрим монокристаллы $\mathrm{AlN}$ и $\mathrm{GaN}$ и оценим параметры, входящие в выражения (2) и (3). Так как $Z^{*} \propto \sqrt{\varepsilon_{0} M\left(\omega_{\mathrm{LO}}^{2}(0)-\omega_{\mathrm{TO}}^{2}(0)\right)}[11]$, где $\varepsilon_{0}-$ статическая диэлектрическая проницаемость, то, воспользовавшись результатами работ [12] $\left(\omega_{\mathrm{TO}}(0)=611 \mathrm{~cm}^{-1}\right.$ для $\mathrm{AlN}$ и $532 \mathrm{~cm}^{-1}$ для $\left.\mathrm{GaN}\right)$ и [13] $\left(\varepsilon_{0}=8.5\right.$ для $\mathrm{AlN}$ и 8.9 для $\mathrm{GaN})$ и учитывая, что для $\mathrm{GaN}$ рассчитанный из первых принципов заряд Борна $Z^{*}=2.2$ [8], для AlN получим $Z^{*}=2.4$ (в [14] для кубического AlN приводится 
значение 2.36). Геометрические параметры элементарных ячеек приведены в [15-17]. Для оценки величины

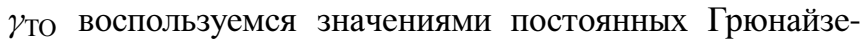
на $\gamma_{\text {TO }}=0.92$ и 0.87 для $\mathrm{AlN}$ и $\mathrm{GaN}[18]$. Функция $C_{V}(T)$ для $\mathrm{GaN}$, приведенная на рис. 1 работы $[10]$, практически совпадает с аппроксимацией теплоемкости решетки при постоянном давлении $C_{P}(T)=(35.6$ $\left.+9.32 \cdot 10^{-3} T\right) 10^{-5} \mathrm{eV} / \mathrm{K}$ при $298<T(K)<1773[13]$. Для $\mathrm{AlN} C_{P}(T)=\left(47.8+3.48 \cdot 10^{-3} T\right) 10^{-5} \mathrm{eV} / \mathrm{K}$ при $300<T(K)<1800$ [13] (для полуколичественных оценок пренебрежем различием между $C_{V}$ и $\left.C_{P}\right)$. Используя найденные значения параметров, получим при комнатной температуре $u_{T}=1.8 \cdot 10^{-6} \mathrm{~K}^{-1}$ для AlN и $1.4 \cdot 10^{-6} \mathrm{~K}^{-1}$ для GaN. Таким образом, $u_{T}(\mathrm{GaN}) / u_{T}(\mathrm{AlN}) \approx C_{P}(\mathrm{GaN}) / C_{P}(\mathrm{AlN}) \approx 0.8$. Отметим, что значения $M \omega_{\text {TO }}^{2}$ определяются силовыми константами, практически одинаковыми для AlN и $\mathrm{GaN}$ вследствие близости постоянных решетки $a$ и $c$.

Подставляя найденные значения $u_{T}$ в формулу (2), получим $p_{1}^{(1)}=1.4 \mu \mathrm{C} / \mathrm{m}^{2} \cdot \mathrm{K}$ для $\mathrm{AlN}$ и $1.2 \mu \mathrm{C} / \mathrm{m}^{2} \cdot \mathrm{K}$ для $\mathrm{GaN}$. C учетом сделанных приближений, полученные результаты вполне удовлетворительно согласуются с результатами расчетов из первых принципов: $-p_{1}^{(1)}=0.9$ и $\sim 1.4 \mu \mathrm{C} / \mathrm{m}^{2} \cdot \mathrm{K}$ при $T=300 \mathrm{~K}$ и $1000 \mathrm{~K}$ для $\mathrm{AlN}[10] ;-p_{1}^{(1)} \cong 1.8 \mu \mathrm{C} / \mathrm{m}^{2} \cdot \mathrm{K}$ при $T=(300-1000) \mathrm{K}$ для $\mathrm{GaN}[8]$ (символ $\sim$ означает, что значения РС сняты с графиков). (Отрицательные значения, приписываемые традиционно коэффициентам $p_{1}^{(1,2)}, p_{2}$ и $p$, не следуют непосредственно из формул (1)-(4), а лишь указывают на то, что спонтанная поляризация $P_{s}$ считается отрицательной. Дело в том, что дипольный момент межатомной связи, вытянутой вдоль оси $c$, направлен от аниона к катиону, т.е. в сторону, противоположную направлению оси $c$.) С другой стороны, несколько удивляет значительное количественное расхождение результатов работ [8] и [10]. Отметим также, что расчеты работы [9] показали, что для высоких температур вклад $\left|p_{1}^{(2)}\right|$ в $\left|p_{1}\right|$ в два раза превышает вклад $\left|p_{1}^{(1)}\right|$ (результат, вызвавший удивление у авторов расчета).

Как уже отмечалось выше, РС $p_{2}=\sum d_{i j k} c_{j k l m} \alpha_{l m}$, откуда для структуры вюрцита имеем [10]:

$$
p_{2}=2 e_{31} \alpha_{1}+e_{33} \alpha_{3},
$$

где $e_{i j}$ - пьезоэлектрические константы [19], $\alpha_{i}-$ коэффициенты анизотропного теплового расширения [18]. Зависимости $p_{2}(T) \propto T$ представлены в $[8,9]$ и [10] для $\mathrm{GaN}$ и $\mathrm{AlN}$ соответственно.

В работах [15-17] исследовалась спонтанная поляризация тройных соединений, образованных бинарными соединениями $\mathrm{AlN}, \mathrm{GaN}$ и $\mathrm{InN}$. Для AlN и $\mathrm{GaN}$ были получены значения $P_{s}=-8.70 \cdot 10^{-2} \mathrm{C} / \mathrm{m}^{2}$ и $P_{s}=-3.73 \cdot 10^{-2} \mathrm{C} / \mathrm{m}^{2} \quad[15], \quad$ так что для $\mathrm{Al}_{x} \mathrm{Ga}_{1-x} \mathrm{~N}\left|P_{s}(x)\right| \propto x$. Полученные нами результаты позволяют предположить, что $\mathrm{PC} \mathrm{Al}_{x} \mathrm{Ga}_{1-x} \mathrm{~N}$ также являются линейными функциями состава.
3. До сих пор мы рассматривали монокристаллы, а сейчас обратимся к пироэлектрическим характеристикам более сложных структур, для чего, естественно, придется прибегать к дополнительным упрощениям. Начнем с тонких пленок, сформированных на массивных твердотельных подложках, для которых феноменологическая теория пироэлектрического эффекта была разработана в [20]. Согласно этой теории, РС тонкой пленки

$$
\bar{p}=p_{1}+\bar{p}_{2}, \quad \bar{p}_{2}=p_{2}+2 \bar{e}_{31}\left(\alpha_{\text {sub }}-\bar{\alpha}\right),
$$

где $\alpha_{\text {sub }}$ и $\bar{\alpha}-$ коэффициенты теплового расширения подложки и тонкой пленки, $\bar{e}_{31}-$ пьезоэлектрическая константа тонкой пленки. В работах [21,22], где рассматривались пленки AlN на кремниевой подложке, получены значения $\mathrm{PC}$, равные соответственно $\bar{p}=4.8$ и $6-8 \mu \mathrm{C} / \mathrm{m}^{2} \cdot \mathrm{K}$. В [23] для соединения $\mathrm{Al}_{1-x} \mathrm{Sc}_{x} \mathrm{~N}$ найдено (в ед. $\mu \mathrm{C} / \mathrm{m}^{2} \cdot \mathrm{K}$ ): $\bar{p}=5.46+15 x$ для $x<0.35$, причем $\bar{p}$ не зависит от температуры в интервале $20-80^{\circ} \mathrm{C}$. Таким образом, для тонких пленок РС положителен, так как для структур вюрцита $e_{31}<0[14,20], \alpha_{\mathrm{Si}}<\alpha_{\mathrm{AIN}}[18,24,25]$ и $\bar{p}=p_{1}+\bar{p}_{2}$, $\bar{p}_{2}=p_{2}+2 \bar{e}_{31}\left(\alpha_{\text {sub }}-\bar{\alpha}\right)>|p|$.

Обсудим теперь результаты работы [26], где исследовались диэлектрические и пироэлектрические свойства структур на основе соединений $\mathrm{AlN}$ и $\mathrm{GaN}$, выращенных методом хлорид-гибридной эпитаксии на подложке $\mathrm{SiC} /(111) \mathrm{Si}$, и где получены высокие значения $\mathrm{PC}$, лежащие в диапазоне $(9-18) \mu \mathrm{C} / \mathrm{m}^{2} \cdot \mathrm{K}$, причем максимальное значение наблюдается для структуры $\mathrm{AlN} / \mathrm{Al}_{x} \mathrm{Ga}_{1-x} \mathrm{~N}$ при отношени $\mathrm{Al} / \mathrm{N}$, равном 50.9/49.1.

По нашему мнению, в данном случае следует говорить об эффективном PC $p_{\text {eff, }}$ так как области стехиометрического состава $\mathrm{Al}_{0.5} \mathrm{Ga}_{0.5} \mathrm{~N}$ представляют собой тонкие прослойки, расположенные внутри слоев $\mathrm{Al}_{x} \mathrm{Ga}_{1-x} \mathrm{~N}$. (При этом напрашивается аналогия с электропроводностью, когда вместо удельной проводимости, характеризующей однородный образец, приходится говорить о кондактансе конкретной структуры.) В [26] такая структура рассматривается как композитный материал, содержащий гетеропереходы между областями с различными концентрациями алюминия и галлия. Следует отметить, что подобные, но химически однородные полосчатые структуры (сверхрешетки) были ранее обнаружены в эпитаксиальных пленках карбида кремния $[27,28]$.

Задача о РС композита для сегнетоэлектриков рассматривалась в работе [29]. При этом эффективный РС композита выражался в виде функции РС и диэлектрических проницаемостей составляющих композит соединений и эффективной диэлектрической проницаемостью композита. Согласно такой схеме, $p_{\text {eff }}(x)=x \bar{p}_{\mathrm{AIN}}+(1-x) \bar{p}_{\mathrm{GaN}}$, где, как и выше, черта вверху относится к характеристике эпитаксиального слоя. Такое выражение, однако, не объясняет наличие $p_{\mathrm{eff}}^{\max }=18 \mu \mathrm{C} / \mathrm{m}^{2}$. Более того, высокие значения РС невозможно описать исключительно влиянием подложки, используя выражение (5). Поэтому, вслед 
за авторами работы [26], следует предположить, что основными объектами, определяющими величину РС, являются гетероконтакты. Роль спонтанной поляризации $P_{s}$ гексагональных политипов $\mathrm{NH}-\mathrm{SiC}$ в формировании энергетической диаграммы гетеропереходов $3 \mathrm{C}-\mathrm{SiC} / \mathrm{NH}-\mathrm{SiC} / 3 \mathrm{C}-\mathrm{SiC}$, где кубический политип $3 \mathrm{C}-\mathrm{SiC}$ не обладает спонтанной поляризацией, рассматривалась в работе [30]. Для описания зонной диаграммы композита, построенного из обладающих спонтанной поляризацией гексагональных нитридов галлия и алюминия, в модели [30] требуется заменить $P_{s}$ на разность $P_{s}(\mathrm{AlN})-P_{s}(\mathrm{GaN})$. При этом вариация $T$ ведет к изменению зонной диаграммы, глубин квантовых ям на гетероконтактах и заселенности их квазиуровней. Задача, таким образом, становится достаточно сложной и самосогласованной. Для правильной постановки такой задачи и ее решения требуются, в первую очередь дополнительные экспериментальные исследования.

\section{Благодарности}

Автор признателен А.А. Лебедеву и С.А. Кукушкину за полезные обсуждения.

\section{Конфликт интересов}

Автор заявляет об отсутствии конфликта интересов.

\section{Список литературы}

[1] S.B. Lang. Phys. Today 58, 8, 31 (2005).

[2] R.W. Whatmore. Rep. Prog. Phys. 49, 12, 1335 (1986).

[3] А.К. Таганцев. УФН 152, 7, 423 (1987).

[4] X. Li, S.-G. Lu, X.-Z. Chen, H. Gu, X. Qian, Q.M. Zhang. J. Mater. Chem. C 1, 1, 23 (2013).

[5] M. Born. Rev. Mod. Phys. 17, 2-3, 245 (1945).

[6] B. Szigeti. Phys. Rev. Lett. 35, 22, 1532 (1975).

[7] W.S. Yan, R. Zhang, Z.L. Xie, X.Q. Xiu, Y.D. Zheng, Z.G. Liu, S. Xu, Z.H. He. Appl. Phys. Lett. 94, 24, 242111 (2009).

[8] J. Liu, M.V. Fernandez-Serra, P.B. Allen. Phys. Rev. B 93, 8, 081205(R) (2016).

[9] J. Liu, S.T. Pantelides. Phys. Rev. Lett. 120, 20, 207602 (2018).

[10] W.S. Yan, R. Zhang, X.Q. Xiu, Z.L. Xie, P. Han, R.L. Jiang, S.L. Gu, Y. Shi, Y.D. Zheng. Appl. Phys. Lett. 90, 21, 212102 (2007).

[11] R.M. Martin. Phys. Rev. B 1, 10, 4005 (1970).

[12] V.Yu. Davydov, Yu.E. Kitaev, N. Goncharuk, A.N. Smirnov, J. Graul, O. Semchinova, D. Uffmann, M.B. Smirnov, A.P. Mirgorodsky, R.A. Evarestov. Phys. Rev. B 58, 19, 12899 (1998).

[13] M.E. Levinshtein, S.L. Rumyantsev, M.S. Shur. Properties of Advanced Semiconductor Materials. Wiley, N.Y. (2001).

[14] У. Харрисон. Электронная структура и свойства твердых тел. Мир, М. (1983). Т. 1.

[15] O. Ambacher, J. Majewski, C. Miskys, A. Link, M. Hermann, M. Eickhoff, M. Stutzmann, F. Bernardini, V. Fiorentini, V. Tilak, B. Schaff, L.F. Eastman. J. Phys.: Condens. Matter 14, 13, 3399 (2002).

[16] С.Ю. Давыдов. ФТТ 51, 6, 1161 (2009).
[17] С.Ю. Давыдов, О.В. Посредник. ФТТ 58, 4, 630 (2016).

[18] H. Iwanaga, A. Kunishige, S. Takeuchi. J. Mater. Sci. 35, 10, 2451 (2000).

[19] F. Bernardini, V. Fiorentini, D. Vanderbilt. Phys. Rev. B 56, 16, R10024 (1997).

[20] J.D. Zook, S.T. Liu. J. Appl. Phys. 49, 8, 4604 (1978).

[21] M.-A. Dubois, P. Muralt. Appl. Phys. Lett. 74, 20, 3032 (1999).

[22] V. Fuflyigin, E. Salley, A. Osinsky, P. Norris. Appl. Phys. Lett. 77, 19, 3075 (2000).

[23] N. Kurz, Y. Lu, L. Kirste, M. Reusch, A. Zukauskaite, V. Lebedev, O. Ambacher. Phys. Status Solidi A 215, 13, 1700831 (2018).

[24] H. Tada, A.E. Kumpel, R.E. Lathrop, J.B. Slanina, P. Nieva, P. Zavracky, I.N. Miaoulis, P.Y. Wong. J. Appl. Phys. 87, 9, 4189 (2000).

[25] H. Watanabe, N. Yamada, M. Okaji. Intern. J. Thermophys. 25, 1, 221 (2004).

[26] А.В. Солнышкин, О.Н. Сергеева, О.А. Шустова, Ш.Ш. Шарофидинов, М.В. Старицын, Е.Ю. Каптелов, С.А. Кукушкин, И.П. Пронин. Письма в ЖТФ 47, 9, 7 (2021).

[27] А.А. Лебедев, М.В. Заморянская, С.Ю. Давыдов, Д.А. Кириленко, С.П. Лебедев, Л.М. Сорокин, Д.Б. Шустов, М.П. Щеглов. ФТП 47, 11, 1554 (2013).

[28] А.А. Лебедев, С.Ю. Давыдов, Л.М. Сорокин, Л.В. Шахов. Письма в ЖТФ 41, 23, 89 (2015).

[29] B. Ploss, B. Ploss, F.G. Shin. IEEE Trans. Dielectrics. Electrical Insulation 13, 5, 1170 (2006).

[30] С.Ю. Давыдов, О.В. Посредник. ФТТ 53, 4, 814 (2011).

Редактор Е.В. Толстякова 